\title{
Development of an in-house loop-mediated isothermal amplification (LAMP) assay for detection of Mycobacterium tuberculosis and evaluation in sputum samples of Nepalese patients
}

\section{Correspondence \\ Yasuhiko Suzuki \\ suzuki@czc.hokudai.ac.jp}

Received 8 July 2007

Accepted 9 December 2007

\section{Basu Dev Pandey, ${ }^{1,2}$ Ajay Poudel, ${ }^{3}$ Tomoko Yoda, ${ }^{4}$ Aki Tamaru, ${ }^{4}$ Naozumi Oda, ${ }^{5}$ Yukari Fukushima, ${ }^{6}$ Binod Lekhak, ${ }^{3}$ Basista Risal, ${ }^{3}$ Bishnu Acharya, ${ }^{2}$ Bishwa Sapkota, ${ }^{7}$ Chie Nakajima, ${ }^{6}$ Tooru Taniguchi, ${ }^{8}$ Benjawan Phetsuksiri ${ }^{9}$ and Yasuhiko Suzuki ${ }^{6}$}

\author{
${ }^{1}$ Sukraraj Tropical and Infectious Disease Hospital, Kathmandu, Nepal \\ ${ }^{2}$ Everest International Clinic and Research Center, Kathmandu, Nepal \\ ${ }^{3}$ Department of Microbiology, Tribhuban University, Kathmandu, Nepal \\ ${ }^{4}$ Department of Infectious Diseases, Osaka Prefectural Institute of Public Health, Japan \\ ${ }^{5}$ Asahi Kasei Co. Ltd, Shizuoka, Japan \\ ${ }^{6}$ Research Center for Zoonosis Control, Hokkaido University, Hokkaido, Japan \\ ${ }^{7}$ Department of Research and Biotechnology, Anandaban Hospital, Kathmandu, Nepal \\ ${ }^{8}$ Section of Bacterial Infections, Research Collaboration Center on Emerging and Re-emerging \\ Infections, Osaka University, Nonthaburi, Thailand \\ ${ }^{9}$ National Institute of Health, Department of Medical Sciences, Ministry of Public Health, \\ Nonthaburi, Thailand
}

\begin{abstract}
A number of nucleic acid amplification assays (NAAs) have been employed to detect tubercle bacilli in clinical specimens for tuberculosis (TB) diagnosis. Among these, loop-mediated isothermal amplification (LAMP) is an NAA possessing superior isothermal reaction characteristics. In the present study, a set of six specific primers targeting the Mycobacterium tuberculosis 16S rRNA gene with high sensitivity was selected and a LAMP system (MTB-LAMP) was developed. Using this system, a total of 200 sputum samples from Nepalese patients were investigated. The sensitivity of MTB-LAMP in culture-positive samples was $100 \%$ (96/96), and the specificity in culture-negative samples was $94.2 \%(98 / 104,95 \%$ confidence interval 90.5-97.9\%). The positive and negative predictive values of MTB-LAMP were 94.1 and $100 \%$, respectively. These results indicate that this MTB-LAMP method may prove to be a powerful tool for the early diagnosis of TB.
\end{abstract}

\section{INTRODUCTION}

Tuberculosis (TB), caused by Mycobacterium tuberculosis, is a common human disease, with 5.1 million new cases and 1.6 million deaths worldwide in 2005 (WHO, 2007). Although TB is often associated with developing nations and disadvantaged economic conditions, it is also gaining ground in many industrialized nations due to immigration, the emergence of drug-resistant strains and the AIDS epidemic.

Abbreviations: AFB, acid-fast bacilli; LAMP, loop-mediated isotherma amplification; NAA, nucleic acid amplification assay; TB, tuberculosis.
Among the countries substantially affected by TB, Nepal has an elevated annual risk of infection, with an estimated $45 \%$ of the total population being infected. In 2005, approximately 48000 people suffered from active $\mathrm{TB}$, of whom 22000 had infectious smear-positive pulmonary disease (WHO, 2007). Although the introduction of the Directly Observed Treatment, Short-course (DOTS) method has reduced the number of deaths, over 6000 people in Nepal continue to die of TB every year (NTP, 2004).

Diagnosis of TB involves clinical evaluation, including the tuberculin skin test, chest radiography and bacteriological tests. Currently, bacteriological tests depend on microscopic observation of acid-fast bacilli (AFB) in clinical 
specimens and bacterial cultures from processed sputum samples, which has been the traditional method for many decades (Kent \& Kubica, 1985). Although these techniques have been continuously refined and improved, they still have several limitations. Although sensitivity and specificity are insufficient, microscopic observation of AFB is widely used due to its rapidity. In contrast, bacteriological testing using bacterial cultures is considered the most accurate test due to its high sensitivity and specificity. However, this technique is both labour-intensive and timeconsuming, requiring 6-8 weeks to achieve maximum sensitivity (Kent \& Kubica, 1985). An additional technique, nucleic acid amplification assay (NAA), allows the rapid, sensitive and specific detection of M. tuberculosis in sputum samples by amplifying and detecting specific nucleic acid sequences (CDC, 1992). In addition to the widely used PCR, several other methods of nucleic acid amplification have been used for M. tuberculosis detection (Jonas et al., 1993; Ruiz-Serrano et al., 1998; Iwamoto et al., 2003; Takakura et al., 2005), including loop-mediated isothermal amplification (LAMP), a novel NAA which enables the detection of trace amounts of DNA under isothermal conditions, namely at $64{ }^{\circ} \mathrm{C}$ (Notomi et al., 2000). LAMP showing high amplification efficiency has been used for the diagnosis of several diseases (Notomi et al., 2000; Iwamoto et al., 2003; Kuboki et al., 2003; Parida et al., 2004, 2005; Kimura et al., 2005; Yoda et al., 2007).

Here, we established an M. tuberculosis detection system using LAMP, and compared its sensitivity, specificity and applicability with results from culture, microscopic observation of acid-fast stained smears and chest radiography.

\section{METHODS}

\section{Establishment of a LAMP method for the detection of $M$.} tuberculosis. More than 50 of six primers recognizing eight distinct regions of the $16 \mathrm{~S}$ rRNA gene were designed using the PrimerExplorer V3 software (Eiken Chemical; https://primerexplorer.jp/lamp3.0.0/ index.html). A primer set was composed of outer primers F3 and B3, inner primers FIP and BIP, and loop primers FLP and BLP. From our preliminary experiments, a set was selected based on its high sensitivity. The specificity of the selected primer set was examined by LAMP reaction on extracted DNA from various bacterial strains, including Mycobacterium tuberculosis, Mycobacterium bovis Ravenel, M. bovis BCG, Mycobacterium africanum, Mycobacterium microti, Mycobacterium kansasii, Mycobacterium avium, Mycobacterium intracellulare, Mycobacterium shimoidei, Mycobacterium nonchromogenicum, Mycobacterium xenopi, Mycobacterium scrofulaceum, Mycobacterium gordonae, Mycobacterium chelonae, Mycobacterium fortuitum, Achromobacter xylosoxidans, Acinetobacter haemolyticus, Bacillus cereus, Escherichia coli, Klebsiella pneumoniae, Legionella pneumophila, Pseudomonas aeruginosa, Shigella boydii, Staphylococcus aureus and Streptococcus haemolyticus. Genomic DNA from bacterial strains was prepared from colonies by mechanical disruption as previously described (Suzuki et al., 1995) and dissolved in $300 \mu \mathrm{lTE}$ buffer consisting of $10 \mathrm{mM}$ Tris/ $\mathrm{HCl}(\mathrm{pH} \mathrm{8.0)}$ and $1 \mathrm{mM}$ EDTA. LAMP reactions were performed in a total volume of $25 \mu \mathrm{l}$ consisting of $30 \mathrm{pmol}$ each of inner primers FIP and BIP, 5 pmol each of outer primers $\mathrm{F} 3$ and $\mathrm{B} 3,20 \mathrm{pmol}$ each of loop primers FLP and BLP, $1.4 \mathrm{mM}$ deoxynucleoside triphosphate, $0.8 \mathrm{M}$ betaine, $20 \mathrm{mM}$ Tris/
$\mathrm{HCl}(\mathrm{pH} 8.8), 10 \mathrm{mM} \mathrm{KCl}, 10 \mathrm{mM}\left(\mathrm{NH}_{4}\right)_{2} \mathrm{SO}_{4}, 8 \mathrm{mM} \mathrm{MgSO}_{4}$ and $8 \mathrm{U}$ Bst DNA polymerase (New England Biolabs) with specified amounts of bacterial DNA. The mixture was incubated at $64{ }^{\circ} \mathrm{C}$ for $60 \mathrm{~min}$ in a Loopamp real-time turbidimeter (LA-200; Teramecs). The reaction was considered positive when turbidity was greater than or equal to 0.1 within $60 \mathrm{~min}$. The sensitivity of the primer set was examined by LAMP reaction on serially diluted DNA preparations from cultured M. tuberculosis H37Ra.

In addition, the sensitivity of the primer set was examined using sputum samples from patients. The sediment layer of $N$-acetyl-Lcysteine- $\mathrm{NaOH}$-treated sputum was suspended in distilled water using standard procedures (Kent \& Kubica, 1985) and serially diluted with sterile distilled water to $10^{-1}, 10^{-2}, 10^{-3}, 10^{-4}$ and $10^{-5}$ concentrations. Following this, $0.1 \mathrm{ml}$ of each dilution was cultured on Löwenstein-Jensen (L-J) medium at $37{ }^{\circ} \mathrm{C}$ for 4 weeks to count bacterial colonies. DNA was extracted from the sputum samples using a DNA extraction kit (DNeasy Blood \& Tissue kit; Qiagen) and used for LAMP reaction. Results were visualized with fluorescence detection reagent (Eiken) according to the manufacturer's instructions. The relation between the number of colonies observed on the L-J medium and the MTB-LAMP results was then evaluated to assess sensitivity on sputum samples.

Evaluation of MTB-LAMP using sputum samples. This study was carried out from September 2004 to October 2005 in Nepal. Samples were collected and examined by acid-fast smear and bacterial culture at the National Tuberculosis Center (NTC), DNA extraction was performed at the Mycobacterial Research Laboratory, Anandaban Hospital, and MTB-LAMP reactions were carried out at the Everest International Clinic and Research Center. One hundred AFB-positive sputum samples from pulmonary TB patients and 100 AFB-negative sputum samples from patients with chest pain, cough and fever were collected at the NTC. During sample and data collection, study objectives and expected outcomes were explained to each study participant before receiving their consent. About $4 \mathrm{ml}$ of mucoid or muco-purulent early morning sample was collected, followed on the same day by microscopy and sample culture preparation. A $50 \mu \mathrm{l}$ sputum sample was smeared directly on a slide, covering an area of approximately $2.0 \times 1.0 \mathrm{~cm}$. For the detection of $\mathrm{AFB}$, a standard fluorochrome method (Truant et al., 1962) was employed. Results were interpreted based on WHO guidelines. The remaining sample was treated by conventional methods as above and used for culture growth and MTB-LAMP. For culture growth, $0.1 \mathrm{ml}$ sputum suspension was inoculated into two culture tubes containing $2 \%$ Ogawa medium and incubated at $37{ }^{\circ} \mathrm{C}$. When colonies were observed during incubation, acid-fastness of bacilli was determined by a smear test. When no colonies appeared after weekly observation for 8 weeks, the result was considered negative.

Mycobacterial DNA was extracted from concentrated sputum samples by freezing and boiling (Woods \& Cole, 1989). LAMP reactions were performed as described above using $5 \mu$ l extracted DNA. Results were visualized using fluorescence detection reagent. Patients with lung cavity observed by chest radiography were considered X-ray-positive.

\section{RESULTS AND DISCUSSION}

The diagnosis of $\mathrm{TB}$ relies primarily on microscopic analysis, which is a rapid method of detecting AFB. However, this method is limited in sensitivity and ability to identify infecting species. In contrast, although the bacterial culture method can efficiently identify species, it is a timeconsuming procedure. A test that combines the rapidity of microscopy and sensitivity of bacterial culture methods 
would facilitate the initiation of clinical TB treatment. Here, detection of $M$. tuberculosis using the MTB-LAMP system was developed and evaluated.

\section{Development of LAMP for the detection of M. tuberculosis}

Among more than 50 sets of primers examined during our preliminary study, a set was selected based on its high sensitivity for purified DNA of M. tuberculosis H37Ra. As shown in Fig. 1(a), these primers targeted the first variable region of the rrs gene. Positions and orientations of F3, F2, FL, F1c, B1, BL, B2c and B3c primers (Notomi et al., 2000) are shown by arrows in Fig. 1(b). The sequences of MTBFIP, MTB-BIP, MTB-F3, MTB-B3, MTB-FLP and MTBBLP primers are shown in Fig. 1(c).

We first examined the specificity of MTB-LAMP for genomic DNA from various bacterial species. Efficient DNA amplification by MTB-LAMP was observed in the M. tuberculosis complex including M. tuberculosis, M. bovis,
M. africanum and M. microti (Fig. 2a). In contrast, no DNA amplification was observed with the remaining bacterial species evaluated. This absence of amplification was also observed using larger amounts of bacterial genomic DNA (10 ng) (data not shown). These results suggest that the primer set used for LAMP is highly specific for the $M$. tuberculosis complex. The specificity of MTB-LAMP was further confirmed using DNA from $M$. tuberculosis, the $M$. avium complex and M. kansasii clinical isolates. MTBLAMP reactions were positive for all $121 \mathrm{M}$. tuberculosis samples and negative for all $17 \mathrm{M}$. avium complex and 18 M. kansasii clinical isolates (Table 1). These results clearly demonstrate the high specificity of the LAMP system in detecting $M$. tuberculosis. Further confirmation of the selected primer set sensitivity was obtained by serially diluting DNA from M. tuberculosis H37Ra. Specific DNA amplification by MTB-LAMP was observed with starting DNA amounts as low as $100 \mathrm{fg}$ (Fig. 2b). Because the weight of 4.5 billion base pairs from one bacterial genome is estimated at $5 \mathrm{fg}$, this result suggests that as few as 20

(a)

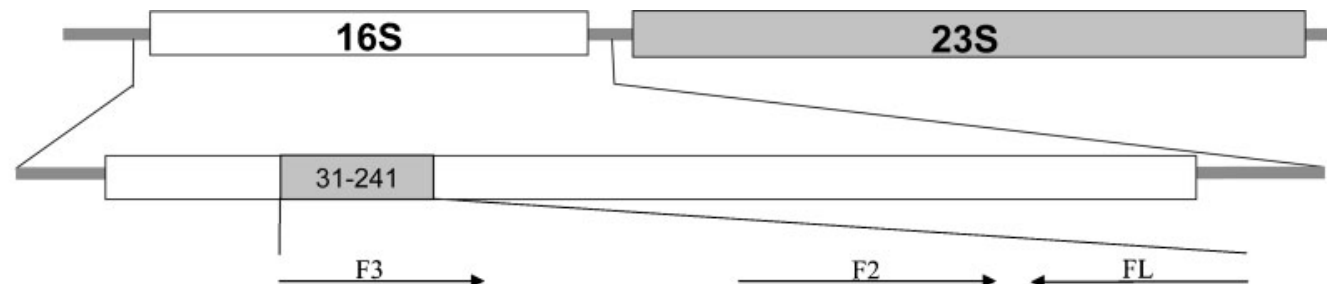

(b)

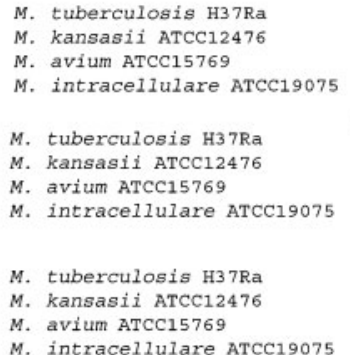

CTGGCTCAGGACGAACGCTGGCGGCGTGCTTAACACATGCAAGTCGAACGGAAAGGTCTCTTCGGAGATACTCGAGTGGC TTGGCTCAGGACGAACGCTGGCGGCGTGCTTAACACATGCAAGTCGAACGGAAAGGTCTCTTCGGAGACACTCGAGTGGC CTGGCTCAGGACGAACGCTGGCGGCGTGCTTAACACATGCAAGTCGAACGGAAAGGCCTCTTCGGAGGTACTCGAGTGGC CTGGCTCAGGACGAACGCTGGCGGCGTGCTTAACACATGCAAGTCGAACGGAAAGGCCTCTTCGGAGGTACTCGAGTGGC $\underset{\text { GAACGGGTGAGTAACACGTGGGTGATCTGCCCTGCACTTCGGGATAAGCCTGGGAAACTGGGTCTAATACCGGATAGGAC }}{\stackrel{\mathrm{Bl}}{\longrightarrow}}$ GAACGGGTGAGTAACACGTGGGCAATCTGCCCTGCACACCGGGATAAGCCTGGGAAACTGGGTCTAATACCGGATAGGAC GAACGGGTGAGTAACACGTGGGCAATCTACCCTGCACTT CGGGATAAGCCTGGGAAACTGGGTCTAATACCGGATAGGAC GAACGGGTGAGTAACACGTGGGCAATCTGCCCTGCACTTCGGGATAAGCCTGGGAAACTGGGTCTAATACCGGATAGGAC $\longleftarrow$ B2C CACTTGGCGCATGCCTTGTGGTGGAAAGC_-TTTI TGCGGTGTGGGATGGGC CTCAAGA_CGCATGTCTTCTTGTGGAAAGC-TTTTGCGGTGTGGGATGĞGC M. intracellulare ATCC19075 CTTTAGGCGCATGTCTTIAGGTGGAAAGC-TTTTECGGTGTGGGATGGGC

(c)

\begin{tabular}{|c|c|c|c|}
\hline Name & & & Sequence \\
\hline MTB-FIP & Flc & F2 & CACCCACGTGTTACTCATGCAAGTCGAACGGAAAGGTCT \\
\hline MTB-BIP & B1 & B2c & TCGGGATAAGCCTGGACCACAAGACATGCATCCCGT \\
\hline MTB-F3 & F3 & & CTGGCTCAGGACGAACG \\
\hline MTB-B3 & $\mathrm{B} 3 \mathrm{c}$ & & GCTCATCCCACACCGC \\
\hline MTB-FLP & $\mathrm{FL}$ & & GTTCGCCACTCGAGTATCTCCG \\
\hline MTB-BLP & $\mathrm{BL}$ & & GAAACTGGGTCTAATACCGG \\
\hline
\end{tabular}

Fig. 1. Primers for the specific detection of the M. tuberculosis complex. (a) Position of the target on the 16S rRNA ( $r r s$ ) gene for MTB-LAMP (near first variable region: 31-241). (b) Position and orientation of the primer annealing site on the first variable region of the rrs gene. Bases different from those in M. tuberculosis H37Ra are underlined. (c) Composition and sequence of six primers used for MTB-LAMP. F1c and F2 in Fig. 1(b) were combined to form the MTB-FIP primer; B1 and B2c in Fig. 1(b) were combined to form the MTB-BIP primer. F3, B3c, FL and BL in Fig. 1(b) were used as MTB-F3, B3, FLP and BLP primers, respectively. 

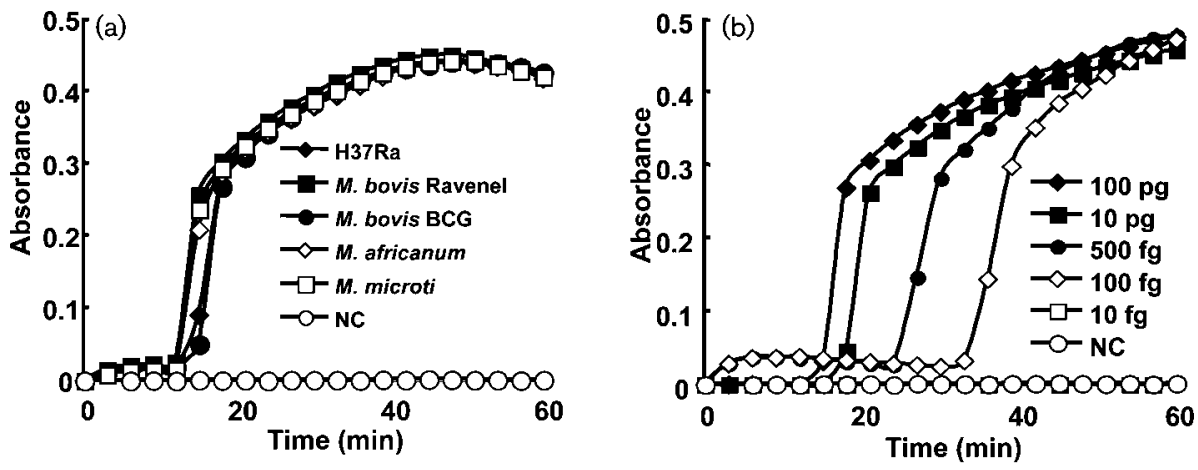

Fig. 2. Specificity and sensitivity of MTB-LAMP determined by real-time turbidimetry. The amount of DNA amplification byproduct (Mg-pyrophosphate) was monitored by measuring turbidity $(590 \mathrm{~nm})$. NC, Negative control. (a) Specificity of MTBLAMP analysed using extracted DNA from various bacterial species. Only results for the M. tuberculosis complex are shown. No positive reaction was observed with DNA from the other bacterial species. (b) Sensitivity of MTB-LAMP analysed using various concentrations of $M$. tuberculosis H37Ra DNA.

copies of M. tuberculosis DNA can be detected using our primer set. Using a bacterial culture of the same $\mathrm{NaOH}$ treated sputum samples, the minimum detectable number of live M. tuberculosis species in a sputum sample by MTBLAMP was determined to be 10 (data not shown). These observations show that MTB-LAMP is more sensitive than a LAMP method used in a previous study, in which the detection of $M$. tuberculosis targeting the gyrB gene was estimated to require a minimum of 50 copies of DNA (Iwamoto et al., 2003).

\section{Evaluation of MTB-LAMP for the detection of $\boldsymbol{M}$. tuberculosis in clinical specimens}

Application of MTB-LAMP to clinical specimens was evaluated by comparing LAMP results with acid-fast smear tests, bacterial cultures and chest radiographs of 200 patients in Nepal. Results are summarized in Table 2. In culture-positive $\left(\mathrm{C}^{+}\right)$samples, $M$. tuberculosis was detected by MTB-LAMP in all 90 smear-positive $\left(\mathrm{S}^{+}\right)$and 6 smearnegative $\left(\mathrm{S}^{-}\right)$samples. The sensitivity of MTB-LAMP in $\mathrm{C}^{+}$specimens was $100 \%(96 / 96)$. This result suggests that MTB-LAMP may be superior to the acid-fast smear test and comparable with bacterial culture testing, though the number of $\mathrm{C}^{+}$and $\mathrm{S}^{-}$samples was not large enough to

Table 1. Reactivity of MTB-LAMP with $10 \mathrm{ng}$ DNA from $M$. tuberculosis, M. avium complex and $M$. kansasii clinical isolates

\begin{tabular}{|lcc|}
\hline \multirow{2}{*}{ Clinical isolates } & \multicolumn{2}{c|}{ LAMP reaction } \\
\cline { 2 - 3 } & + & - \\
\hline M. tuberculosis & $121 / 121$ & $0 / 121$ \\
M. avium complex & $0 / 17$ & $17 / 17$ \\
M. kansasii & $0 / 18$ & $18 / 18$ \\
\hline
\end{tabular}

strongly conclude this. In culture-negative $\left(\mathrm{C}^{-}\right)$samples, MTB-LAMP reactions were positive in 1 of $5 \mathrm{~S}^{+}$X-raynegative $\left(\mathrm{X}^{-}\right)$cases; 2 of $13 \mathrm{~S}^{-} \mathrm{X}^{+}$cases; and in 3 of $81 \mathrm{~S}^{-}$ $\mathrm{X}^{-}$cases. No positive MTB-LAMP reaction was observed in the five $\mathrm{S}^{+} \mathrm{X}^{+}$patients. MTB-LAMP specificity in $\mathrm{C}^{-}$ samples was $94.2 \%(98 / 104 ; 95 \%$ confidence interval $90.5-97.9 \%)$. The positive and negative predictive values of MTB-LAMP were 94.1 and $100 \%$, respectively. These results from $\mathrm{C}^{-}$patients may suggest that MTB-LAMP is possibly highly sensitive in the detection of a small number of tubercle bacilli released from hidden foci in sputum.

M. tuberculosis was detected by MTB-LAMP in all $\mathrm{C}^{+}$and a number of $\mathrm{C}^{-}$samples. Compared with results from standard culture testing, acid-fast staining and chest radiography, our results demonstrate the high clinical performance of MTB-LAMP in the rapid detection of M. tuberculosis from sputum samples. A study by Iwamoto et al. (2003) on LAMP sensitivity in sputum samples indicated that the detection limit of their LAMP assay is equivalent to that of the Amplicor assay (Roche Diagnostics). In some $\mathrm{C}^{+}$sputum samples, however, their system was unable to detect $M$. tuberculosis. In contrast, the sensitivity of our MTB-LAMP appears to be superior

Table 2. Comparison of MTB-LAMP results with smear, culture and chest radiograph results

The table shows the percentage of M. tuberculosis LAMP-positive cases (no. of positive/total cases).

\begin{tabular}{|lcrlcc|}
\hline & \multicolumn{2}{c}{ Culture (+) } & & \multicolumn{2}{c|}{ Culture (-) } \\
\cline { 2 - 3 } & X-ray (+) & X-ray (-) & & X-ray (+) & X-ray (-) \\
\hline Smear (+) & $100 \%(90 / 90)$ & $0 \%(0 / 0)$ & $0 \%(0 / 5)$ & $20 \%(1 / 5)$ \\
Smear (-) & $100 \%(5 / 5)$ & $100 \%(1 / 1)$ & $15 \%(2 / 13)$ & $3.7 \%(3 / 81)$ \\
\hline & $100 \%(96 / 96)$ & & $5.8 \%(6 / 104)$ \\
\hline
\end{tabular}


because of the detection of $M$. tuberculosis in all $96 \mathrm{C}^{+}$ samples ( $100 \%$ sensitivity). A multicentre study on $M$. tuberculosis detection showed the feasibility of using the LAMP method in developing countries (Boehme et al., 2007). The study evaluated a prototype LAMP assay targeting the $g y r B$ gene and using a simplified manual DNA extraction method. The sensitivity of MTB-LAMP was $97.7 \%(173 / 177)$ in $\mathrm{S}^{+}$and $\mathrm{C}^{+}$sputum samples and $48.8 \%(21 / 43)$ in $\mathrm{S}^{-}$and $\mathrm{C}^{+}$sputum samples. This is lower than in the present results, with a sensitivity of $100 \%$ (90/90) and $100 \%(6 / 6)$ for $\mathrm{C}^{+} \mathrm{S}^{+}$and $\mathrm{S}^{-}$sputum samples, respectively. Although our MTB-LAMP method is more time-consuming regarding sputum sample manipulation, it has the advantage of being highly sensitive.

In conclusion, this MTB-LAMP assay is an NAA method that allows direct identification of $M$. tuberculosis in processed sputum samples. Due to its ease of use in developing countries and its high sensitivity and specificity, this assay may facilitate the identification of $M$. tuberculosis in $\mathrm{S}^{+}$cases, as well as the confirmation of diagnosis in suspected but sputum $\mathrm{S}^{-}$clinical TB cases.

\section{ACKNOWLEDGEMENTS}

We gratefully thank Dr M. Mondro and Mr Chaman Shrestha from the Department of Research and Biotechnology, Anandaba Leprosy Hospital, Nepal, as well as Mr Kiran Pandey at the Everest International Clinic and Research Center, Kathmandu, Nepal, for their technical help. We would also like to thank the Director and staff of the Nepal Tuberculosis Center for providing the culture facility. This work was supported in part by Grants-in-Aid for the Program of Founding Research Center for Emerging and Reemerging Infectious Diseases from the Ministry of Education, Culture, Sports, Science, and Technology, Japan, to Y.S.

\section{REFERENCES}

Boehme, C. C., Nabeta, P., Henostroza, G., Raqib, R., Rahim, Z., Gerhardt, M., Sanga, E., Hoelscher, M., Notomi, T. \& other authors (2007). Operational feasibility of using loop-mediated isothermal amplification for diagnosis of pulmonary tuberculosis in microscopy centers of developing countries. J Clin Microbiol 45, 1936-1940.

Centers for Disease Control and Prevention (1992). National action plan to combat multidrug-resistant tuberculosis: summary of a conference; management of persons exposed to multidrug-resistant tuberculosis. Morbid Mortal Weekly Rep 41, 5-48.

Iwamoto, T., Sonobe, T. \& Hayashi, K. (2003). Loop-mediated isothermal amplification for direct detection of Mycobacterium tuberculosis complex, M. avium, and M. intracellulare. J Clin Microbiol 41, 2616-2622.

Jonas, V., Alden, M. J., Curry, J. I., Kamisango, K., Knott, C. A., Lankford, R., Wolfe, J. M. \& Moore, D. F. (1993). Detection and identification of Mycobacterium tuberculosis directly from sputum sediments by amplification of rRNA. J Clin Microbiol 31, 2410-2416.

Kent, B. D. \& Kubica, G. P. (1985). Public Health Mycobacteriology: a Guide for the Level III Laboratory. Atlanta: US Department of Health and Human Services, Centers for Disease Control.

Kimura, H., Ihira, M., Enomoto, Y., Kawada, J., Ito, J., Morishima, T., Yoshikawa, T. \& Asano, Y. (2005). Rapid detection of herpes simplex virus DNA in cerebrospinal fluid: comparison between loop-mediated isothermal amplification and real-time PCR. Med Microbiol Immunol 194, 181-185.

Kuboki, N., Inoue, N., Sakurai, T., Cello, F. D., Grab, D. J., Suzuki, H., Sugimoto, C. \& Igarashi, I. (2003). Loop-mediated isothermal amplification for detection of African trypanosomes. J Clin Microbiol 41, 5517-5524.

Notomi, T., Okayama, H., Masubuchi, H., Yonekawa, T., Watanabe, K. \& Amino, N. (2000). Loop-mediated isothermal amplification of DNA. Nucleic Acids Res 28, E63.

NTP (2004). Annual report of National Tuberculosis Control Programme Nepal. His Majesty's Government of Nepal, Ministry of Health, Department of Health Services.

Parida, M., Inoue, G. P. S., Hasebe, F. \& Morita, K. (2004). Real-time reverse transcription loop-mediated isothermal amplification for rapid detection of West Nile virus. J Clin Microbiol 42, 257-263.

Parida, M., Horioke, K., Ishida, H., Dash, P. K., Saxena, P., Jana, A. M., Islam, M. A., Inoue, S., Hosaka, N. \& Morita, K. (2005). Rapid detection and differentiation of dengue virus serotypes by a real-time reverse transcription-loop-mediated isothermal amplification assay. $J$ Clin Microbiol 43, 2895-2903.

Ruiz-Serrano, M. J., Albadalejo, J., Martinez-Sanchez, L. \& Bouza, E. (1998). LCx: a diagnostic alternative for the early detection of Mycobacterium tuberculosis complex. Diagn Microbiol Infect Dis 32, 259-264.

Suzuki, Y., Katsukawa, C., Inoue, K., Yin, Y. P., Tasaka, H., Ueba, N. \& Makino, M. (1995). Mutations in rpoB gene of rifampicin resistant clinical isolates of Mycobacterium tuberculosis in Japan. Kansenshogaku Zasshi 69, 413-419.

Takakura, S., Tsuchiya, S., Isawa, Y., Yasukawa, K., Hayashi, T., Tomita, M., Suzuki, K., Hasegawa, T., Tagami, T. \& other authors (2005). Rapid detection of Mycobacterium tuberculosis in respiratory samples by transcription-reverse transcription concerted reaction with an automated system. J Clin Microbiol 43, 5435-5439.

Truant, J. P., Brett, W. A. \& Thomas, W., Jr (1962). Fluorescence microscopy of tubercle bacilli stained with auramine and rhodamine. Henry Ford Hosp Med Bull 10, 287-296.

WHO (2007). Global Tuberculosis Control: Surveillance, Planning, Financing. WHO report (document WHO/HTM/TB/2005, 349). Geneva: World Health Organization.

Woods, S. A. \& Cole, S. T. (1989). A rapid method for the detection of potentially viable Mycobacterium leprae in human biopsies: a novel application of PCR. FEMS Microbiol Lett 53, 305-309.

Yoda, T., Suzuki, Y., Yamazaki, K., Sakon, N., Kanki, M., Aoyama, I. \& Tsukamoto, T. (2007). Evaluation and application of reverse transcription loop-mediated isothermal amplification for detection of noroviruses. J Med Virol 79, 326-334. 